\title{
A CAGINALP PHASE-FIELD SYSTEM BASED ON TYPE III HEAT CONDUCTION WITH TWO TEMPERATURES
}

\author{
ALAIN MIRANVILLE ${ }^{1}$, RAMON QUINTANILLA ${ }^{2}$
}

\begin{abstract}
Our aim in this paper is to study a generalization of the Caginalp phasefield system based on the theory of type III thermomechanics with two temperatures for the heat conduction. In particular, we obtain well-posedness results and study the dissipativity of the associated solution operators. We consider here both regular and singular nonlinear terms. Furthermore, we endow the equations with two types of boundary conditions, namely, Dirichlet and Neumann. Finally, we study the spatial behavior of the solutions in a semi-infinite cylinder, when such solutions exist.
\end{abstract}

\section{INTRODUCTION}

The Caginalp phase-field system,

$$
\frac{\partial u}{\partial t}-\Delta u+f(u)=T
$$

$$
\frac{\partial T}{\partial t}-\Delta T=-\frac{\partial u}{\partial t}
$$

has been proposed in [5] to model phase transition phenomena, such as melting-solidification phenomena. Here, $u$ is the order parameter, $T$ is the relative temperature (defined as $T=\tilde{T}-T_{E}$, where $\tilde{T}$ is the absolute temperature and $T_{E}$ is the equilibrium melting temperature) and $f$ is the derivative of a double-well potential $F$. Furthermore, here and below, we set all physical parameters equal to one. This system has been much studied; we refer the reader to, e.g., [1], [2], [3], [4], [9], [10], [11], [18], [20], [21], [22], [23], [24], [32], [39] and [45].

These equations can be derived as follows. One introduces the (total Ginzburg-Landau) free energy

$$
\Psi=\int_{\Omega}\left(\frac{1}{2}|\nabla u|^{2}+F(u)-u T-\frac{1}{2} T^{2}\right) d x
$$

where $\Omega$ is the domain occupied by the system (we assume here that it is a bounded and regular domain of $\mathbb{R}^{n}, n=1,2$ or 3 , with boundary $\Gamma$ ), and the enthalpy

$$
H=u+T \text {. }
$$

2010 Mathematics Subject Classification. 35K55, 35J60, 80A22.

Key words and phrases. Caginalp system, Type III thermomechanics, two temperatures, wellposedness, dissipativity, spatial behavior, Phragmén-Lindelöf alternative. 
As far as the evolution equation for the order parameter is concerned, one postulates the relaxation dynamics (with relaxation parameter set equal to one)

$$
\frac{\partial u}{\partial t}=-\frac{D \Psi}{D u}
$$

where $\frac{D}{D u}$ denotes a variational derivative with respect to $u$, which yields (1.1). Then, we have the energy equation

$$
\frac{\partial H}{\partial t}=-\operatorname{div} q
$$

where $q$ is the heat flux. Assuming finally the usual Fourier law for heat conduction,

$$
q=-\nabla T
$$

we obtain (1.2).

Now, one essential drawback of the Fourier law is that it predicts that thermal signals propagate at an infinite speed, which violates causality (the so-called paradox of heat conduction, see [13]). To overcome this drawback, or at least to account for more realistic features, several alternatives to the Fourier law, based, e.g., on the Maxwell-Cattaneo law or recent laws from thermomechanics, have been proposed and studied, in the context of the Caginalp phase-field system, in [29], [30], [33], [34], [35], [36] and [38].

In the late 1960's, several authors proposed a heat conduction theory based on two temperatures (see [6], [7] and [8]). More precisely, one now considers the conductive temperature $T$ and the thermodynamic temperature $\theta$. In particular, for simple materials, these two temperatures are shown to coincide. However, for non-simple materials, they differ and are related as follows:

$$
\theta=T-\Delta T \text {. }
$$

The Caginalp system, based on this two temperatures theory and the usual Fourier law, was studied in [14].

Our aim in this paper is to study a variant of the Caginalp phase-field system based on the type III thermomechanics theory (see [25]) with two temperatures recently proposed in [43] (see also [15]).

In that case, the free energy reads, in terms of the (relative) thermodynamic temperature $\theta$,

$$
\Psi=\int_{\Omega}\left(\frac{1}{2}|\nabla u|^{2}+F(u)-u \theta-\frac{1}{2} \theta^{2}\right) d x
$$

and (1.5) yields, in view of (1.8), the following evolution equation for the order parameter:

$$
\frac{\partial u}{\partial t}-\Delta u+f(u)=T-\Delta T
$$

Furthermore, the enthalpy now reads 


$$
H=u+\theta=u+T-\Delta T
$$

which yields, owing to (1.6), the energy equation

$$
\frac{\partial T}{\partial t}-\Delta \frac{\partial T}{\partial t}+\operatorname{div} q=-\frac{\partial u}{\partial t} .
$$

Finally, the heat flux is given, in the type III theory with two temperatures, by (see [43])

$$
q=-\nabla \alpha-\nabla T
$$

where

$$
\alpha(t, x)=\int_{0}^{t} T(\tau, x) d \tau+\alpha_{0}(x)
$$

is the conductive thermal displacement. Noting that $T=\frac{\partial \alpha}{\partial t}$, we finally deduce from (1.10) and (1.12)-(1.13) the following variant of the Caginalp phase-field system:

$$
\begin{gathered}
\frac{\partial u}{\partial t}-\Delta u+f(u)=\frac{\partial \alpha}{\partial t}-\Delta \frac{\partial \alpha}{\partial t} \\
\frac{\partial^{2} \alpha}{\partial t^{2}}-\Delta \frac{\partial^{2} \alpha}{\partial t^{2}}-\Delta \frac{\partial \alpha}{\partial t}-\Delta \alpha=-\frac{\partial u}{\partial t} .
\end{gathered}
$$

We can note that we still have an infinite speed of propagation here, since (1.15) is parabolic; actually, also (1.16) is not hyperbolic and we have not been able to prove whether or not it exhibits a finite speed of propagation (note however that, as far as the equation

$$
\frac{\partial^{2} \alpha}{\partial t^{2}}-\Delta \frac{\partial \alpha}{\partial t}-\Delta \alpha=0
$$

known as the strongly damped wave equation, is concerned, one does not have a finite speed of propagation).

Our aim in this paper is to study the well-posedness and the dissipativity of (1.15)(1.16). We consider here two types of boundary conditions, namely, Dirichlet and Neumann. Furthermore, we consider regular nonlinear terms $f$ (a usual choice being the cubic nonlinear term $f(s)=s^{3}-s$ ), as well as singular nonlinear terms (and, in particular, the thermodynamically relevant logarithmic nonlinear terms $f(s)=k_{1} s+\frac{k_{2}}{2} \ln \frac{1+s}{1-s}$, $\left.s \in(-1,1), 0<k_{2}<k_{1}\right)$.

We are also interested in the study of the spatial behavior of the solutions. Spatial decay estimates for partial differential equations are related to the Saint-Venant principle which is both a mathematical and a thermomechanical aspect which has deserved much attention in the last years (see [26] and the references therein). Such studies describe how the influence of the perturbations on a part of the boundary is damped for the points which are far away from the perturbations. Spatial decay estimates for elliptic [16], parabolic 
[27], [28], hyperbolic [17] and/or combinations of such [42] have been obtained in the last years. However, as far as nonlinear equations are concerned, such a knowledge is limited (see [33], [34], [35], [37] and [38]). What is usual is to consider a semi-infinite cylinder whose finite end is perturbed and see what happens when the spatial variable goes to infinity. However, we do not study the existence of solutions to this problem; in fact, this does not seem to be an easy task (see, e.g., [37]). We thus assume the existence of solutions and then only study the spatial asymptotic behavior in that case. More precisely, we obtain a Phragmén-Lindelöf alternative, i.e., either a growth or a decay estimate. An upper bound on the amplitude term, when the solution decays, is also derived, in terms of the boundary conditions.

Notation. We denote by $((\cdot, \cdot))$ the usual $L^{2}$-scalar product, with associated norm $\|\cdot\|$; more generally, $\|\cdot\|_{X}$ denotes the norm on the Banach space $X$.

We set, for $v \in L^{1}(\Omega)$,

and $\bar{v}=v-\langle v\rangle$.

$$
\langle v\rangle=\frac{1}{\operatorname{Vol}(\Omega)} \int_{\Omega} v d x
$$

We further note that

and

$$
v \mapsto\left(\|\bar{v}\|^{2}+\langle v\rangle^{2}\right)^{\frac{1}{2}}
$$

$$
v \mapsto\left(\|\nabla v\|^{2}+\langle v\rangle^{2}\right)^{\frac{1}{2}}
$$

are norms on $L^{2}(\Omega)$ and $H^{1}(\Omega)$, respectively, which are equivalent to their usual norms.

Throughout the paper, the same letters $c$ and $c^{\prime}$ denote (generally positive) constants which may vary from line to line. Similarly, the same letter $Q$ denotes (positive) monotone increasing (with respect to each argument) functions which may vary from line to line.

\section{DiRICHLET BOUNDARY CONDITIONS}

2.1. Setting of the problem. We consider the following initial and boundary value problem:

$$
\begin{gathered}
\frac{\partial u}{\partial t}-\Delta u+f(u)=\frac{\partial \alpha}{\partial t}-\Delta \frac{\partial \alpha}{\partial t} \\
\frac{\partial^{2} \alpha}{\partial t^{2}}-\Delta \frac{\partial^{2} \alpha}{\partial t^{2}}-\Delta \frac{\partial \alpha}{\partial t}-\Delta \alpha=-\frac{\partial u}{\partial t} \\
u=\alpha=0 \text { on } \Gamma \\
\left.u\right|_{t=0}=u_{0},\left.\quad \alpha\right|_{t=0}=\alpha_{0},\left.\frac{\partial \alpha}{\partial t}\right|_{t=0}=\alpha_{1} .
\end{gathered}
$$

As far as the nonlinear term $f$ is concerned, we assume that 


$$
\begin{gathered}
f \in \mathcal{C}^{1}(\mathbb{R}), f(0)=0, \\
f^{\prime} \geq-c_{0}, c_{0} \geq 0, \\
f(s) s \geq c_{1} F(s)-c_{2} \geq-c_{3}, c_{1}>0, c_{2}, c_{3} \geq 0, s \in \mathbb{R},
\end{gathered}
$$

where $F(s)=\int_{0}^{s} f(\xi) d \xi$. In particular, the usual cubic nonlinear term $f(s)=s^{3}-s$ satisfies these assumptions.

2.2. A priori estimates. The estimates derived in this subsection will be formal, but they can easily be justified within a Galerkin scheme.

We multiply (2.1) by $\frac{\partial u}{\partial t}$ and have, integrating over $\Omega$ and by parts,

$$
\frac{1}{2} \frac{d}{d t}\left(\|\nabla u\|^{2}+2 \int_{\Omega} F(u) d x\right)+\left\|\frac{\partial u}{\partial t}\right\|^{2}=\left(\left(\frac{\partial \alpha}{\partial t}-\Delta \frac{\partial \alpha}{\partial t}, \frac{\partial u}{\partial t}\right)\right) .
$$

We then multiply $(2.2)$ by $\frac{\partial \alpha}{\partial t}-\Delta \frac{\partial \alpha}{\partial t}$ to obtain

$$
\frac{1}{2} \frac{d}{d t}\left(\|\nabla \alpha\|^{2}+\|\Delta \alpha\|^{2}+\left\|\frac{\partial \alpha}{\partial t}-\Delta \frac{\partial \alpha}{\partial t}\right\|^{2}\right)+\left\|\nabla \frac{\partial \alpha}{\partial t}\right\|^{2}+\left\|\Delta \frac{\partial \alpha}{\partial t}\right\|^{2}=-\left(\left(\frac{\partial \alpha}{\partial t}-\Delta \frac{\partial \alpha}{\partial t}, \frac{\partial u}{\partial t}\right)\right) \text {. }
$$

Summing (2.8) and (2.9), we find the differential equality

$$
\frac{d E_{1}}{d t}+2\left\|\frac{\partial u}{\partial t}\right\|^{2}+2\left\|\nabla \frac{\partial \alpha}{\partial t}\right\|^{2}+2\left\|\Delta \frac{\partial \alpha}{\partial t}\right\|^{2}=0
$$

where

$$
E_{1}=\|\nabla u\|^{2}+2 \int_{\Omega} F(u) d x+\|\nabla \alpha\|^{2}+\|\Delta \alpha\|^{2}+\left\|\frac{\partial \alpha}{\partial t}-\Delta \frac{\partial \alpha}{\partial t}\right\|^{2}
$$

satisfies

$$
E_{1} \geq c\left(\|u\|_{H^{1}(\Omega)}^{2}+\|\alpha\|_{H^{2}(\Omega)}^{2}+\left\|\frac{\partial \alpha}{\partial t}\right\|_{H^{2}(\Omega)}^{2}\right)-c^{\prime}, c>0
$$

(note indeed that $\left\|\frac{\partial \alpha}{\partial t}-\Delta \frac{\partial \alpha}{\partial t}\right\|^{2}=\left\|\frac{\partial \alpha}{\partial t}\right\|^{2}+2\left\|\nabla \frac{\partial \alpha}{\partial t}\right\|^{2}+\left\|\Delta \frac{\partial \alpha}{\partial t}\right\|^{2}$ ).

Next, we multiply (2.1) by $u$ and have, owing to (2.7),

$$
\frac{d}{d t}\|u\|^{2}+c\left(\|u\|_{H^{1}(\Omega)}^{2}+2 \int_{\Omega} F(u) d x\right) \leq c^{\prime}\left(\left\|\frac{\partial \alpha}{\partial t}\right\|^{2}+\left\|\Delta \frac{\partial \alpha}{\partial t}\right\|^{2}\right), c>0 .
$$

Multiplying then (2.1) by $-\Delta u$, we obtain, owing to (2.6),

$$
\frac{d}{d t}\|\nabla u\|^{2}+c\|u\|_{H^{2}(\Omega)}^{2} \leq c^{\prime}\left(\|\nabla u\|^{2}+\left\|\frac{\partial \alpha}{\partial t}\right\|^{2}+\left\|\Delta \frac{\partial \alpha}{\partial t}\right\|^{2}\right), c>0 .
$$


Summing finally $(2.10), \delta_{1}$ times (2.13) and $\delta_{2}$ times $(2.14)$, where $\delta_{1}, \delta_{2}>0$ are small enough, we find a differential inequality of the form

$$
\frac{d E_{2}}{d t}+c\left(\|u\|_{H^{2}(\Omega)}^{2}+2 \int_{\Omega} F(u) d x+\left\|\frac{\partial u}{\partial t}\right\|^{2}+\left\|\frac{\partial \alpha}{\partial t}\right\|_{H^{2}(\Omega)}^{2}\right) \leq c^{\prime}, c>0,
$$

where

$$
E_{2}=E_{1}+\delta_{1}\|u\|^{2}+\delta_{2}\|\nabla u\|^{2}
$$

satisfies

$$
E_{2} \geq c\left(\|u\|_{H^{1}(\Omega)}^{2}+\|\alpha\|_{H^{2}(\Omega)}^{2}+\left\|\frac{\partial \alpha}{\partial t}\right\|_{H^{2}(\Omega)}^{2}\right)-c^{\prime}, c>0 .
$$

In a next step, we multiply (2.2) by $-\Delta \alpha$ and have

$$
\begin{gathered}
\frac{d}{d t}\left(\|\Delta \alpha\|^{2}-2\left(\left(\frac{\partial \alpha}{\partial t}, \Delta \alpha\right)\right)+2\left(\left(\Delta \frac{\partial \alpha}{\partial t}, \Delta \alpha\right)\right)\right)+\|\Delta \alpha\|^{2} \\
\leq\left\|\frac{\partial u}{\partial t}\right\|^{2}+2\left\|\nabla \frac{\partial \alpha}{\partial t}\right\|^{2}+2\left\|\Delta \frac{\partial \alpha}{\partial t}\right\|^{2} .
\end{gathered}
$$

Summing (2.15) and $\delta_{3}$ times (2.18), where $\delta_{3}>0$ is small enough, we obtain a differential inequality of the form

$$
\frac{d E_{3}}{d t}+c\left(E_{3}+\|u\|_{H^{2}(\Omega)}^{2}+\left\|\frac{\partial u}{\partial t}\right\|^{2}\right) \leq c^{\prime}, c>0
$$

where

$$
E_{3}=E_{2}+\delta_{3}\left(\|\Delta \alpha\|^{2}-2\left(\left(\frac{\partial \alpha}{\partial t}, \Delta \alpha\right)\right)+2\left(\left(\Delta \frac{\partial \alpha}{\partial t}, \Delta \alpha\right)\right)\right)
$$

satisfies

$$
E_{3} \geq c\left(\|u\|_{H^{1}(\Omega)}^{2}+\|\alpha\|_{H^{2}(\Omega)}^{2}+\left\|\frac{\partial \alpha}{\partial t}\right\|_{H^{2}(\Omega)}^{2}\right)-c^{\prime}, c>0 .
$$

We now differentiate (2.1) with respect to time to find, owing to (2.2),

$$
\frac{\partial}{\partial t} \frac{\partial u}{\partial t}-\Delta \frac{\partial u}{\partial t}+f^{\prime}(u) \frac{\partial u}{\partial t}=-\frac{\partial u}{\partial t}+\Delta \frac{\partial \alpha}{\partial t}+\Delta \alpha,
$$

together with the boundary condition

$$
\frac{\partial u}{\partial t}=0 \text { on } \Gamma .
$$

Multiplying (2.22) by $\frac{\partial u}{\partial t}$, we have, owing to (2.6), 


$$
\frac{d}{d t}\left\|\frac{\partial u}{\partial t}\right\|^{2}+c\left\|\frac{\partial u}{\partial t}\right\|_{H^{1}(\Omega)}^{2} \leq c^{\prime}\left(\left\|\frac{\partial u}{\partial t}\right\|^{2}+\|\alpha\|_{H^{2}(\Omega)}^{2}+\left\|\frac{\partial \alpha}{\partial t}\right\|_{H^{2}(\Omega)}^{2}\right), c>0 .
$$

Summing (2.19) and $\delta_{4}$ times (2.24), where $\delta_{4}>0$ is small enough, we obtain a differential inequality of the form

$$
\frac{d E_{4}}{d t}+c\left(E_{4}+\|u\|_{H^{2}(\Omega)}^{2}+\left\|\frac{\partial u}{\partial t}\right\|_{H^{1}(\Omega)}^{2}\right) \leq c^{\prime}, c>0
$$

where

$$
E_{4}=E_{3}+\delta_{4}\left\|\frac{\partial u}{\partial t}\right\|^{2}
$$

satisfies

$$
E_{4} \geq c\left(\|u\|_{H^{1}(\Omega)}^{2}+\left\|\frac{\partial u}{\partial t}\right\|^{2}+\|\alpha\|_{H^{2}(\Omega)}^{2}+\left\|\frac{\partial \alpha}{\partial t}\right\|_{H^{2}(\Omega)}^{2}\right)-c^{\prime}, c>0 .
$$

We finally rewrite (2.1) as an elliptic equation, for $t$ fixed,

$$
-\Delta u+f(u)=\frac{\partial \alpha}{\partial t}-\Delta \frac{\partial \alpha}{\partial t}-\frac{\partial u}{\partial t}, u=0 \text { on } \Gamma .
$$

Multiplying (2.28) by $-\Delta u$, we find, owing to (2.6) and a classical elliptic regularity result,

$$
\|u(t)\|_{H^{2}(\Omega)}^{2} \leq c E_{4}(t)+c^{\prime}, t \geq 0 .
$$

In particular, it follows from (2.25) and Gronwall's lemma that

$$
E_{4}(t) \leq e^{-c t} Q\left(\left\|u_{0}\right\|_{H^{2}(\Omega)},\left\|\alpha_{0}\right\|_{H^{2}(\Omega)},\left\|\alpha_{1}\right\|_{H^{2}(\Omega)}\right)+c^{\prime}, c>0, t \geq 0,
$$

where we have used the continuous embedding $H^{2}(\Omega) \subset \mathcal{C}(\bar{\Omega})$ to deduce that

$$
\left|\int_{\Omega} F\left(u_{0}\right) d x\right| \leq Q\left(\left\|u_{0}\right\|_{H^{2}(\Omega)}\right)
$$

and the fact that

which yields

$$
\frac{\partial u}{\partial t}(0)=\Delta u_{0}-f\left(u_{0}\right)+\alpha_{1}-\Delta \alpha_{1}
$$

$$
\left\|\frac{\partial u}{\partial t}(0)\right\| \leq Q\left(\left\|u_{0}\right\|_{H^{2}(\Omega)},\left\|\alpha_{1}\right\|_{H^{2}(\Omega)}\right) .
$$

Combining (2.29) and (2.30), we finally find, in view of (2.27),

$$
\|u(t)\|_{H^{2}(\Omega)}^{2}+\left\|\frac{\partial u}{\partial t}(t)\right\|^{2}+\|\alpha(t)\|_{H^{2}(\Omega)}^{2}+\left\|\frac{\partial \alpha}{\partial t}(t)\right\|_{H^{2}(\Omega)}^{2}
$$




$$
\leq e^{-c t} Q\left(\left\|u_{0}\right\|_{H^{2}(\Omega)},\left\|\alpha_{0}\right\|_{H^{2}(\Omega)},\left\|\alpha_{1}\right\|_{H^{2}(\Omega)}\right)+c^{\prime}, c>0, t \geq 0 .
$$

2.3. The dissipative semigroup. We have the

Theorem 2.1. We assume that (2.5)-(2.7) hold. Then, for every $\left(u_{0}, \alpha_{0}, \alpha_{1}\right) \in\left(H^{2}(\Omega) \cap\right.$ $\left.H_{0}^{1}(\Omega)\right)^{3},(2.1)-(2.4)$ possesses a unique solution $\left(u, \alpha, \frac{\partial \alpha}{\partial t}\right)$ such that

and

$$
\left(u, \alpha, \frac{\partial \alpha}{\partial t}\right) \in L^{\infty}\left(\mathbb{R}^{+} ; H^{2}(\Omega) \cap H_{0}^{1}(\Omega)\right)^{3}
$$

$$
\frac{\partial u}{\partial t} \in L^{\infty}\left(\mathbb{R}^{+} ; L^{2}(\Omega)\right) \cap L^{2}\left(0, T ; H_{0}^{1}(\Omega)\right), \forall T>0 .
$$

Proof. The proof of existence is based on the a priori estimates derived in the previous subsection and on, e.g., a standard Galerkin scheme.

Let now $\left(u^{(1)}, \alpha^{(1)}, \frac{\partial \alpha^{(1)}}{\partial t}\right)$ and $\left(u^{(2)}, \alpha^{(2)}, \frac{\partial \alpha^{(2)}}{\partial t}\right)$ be two solutions to (2.1)-(2.3) with initial data $\left(u_{0}^{(1)}, \alpha_{0}^{(1)}, \alpha_{1}^{(1)}\right)$ and $\left(u_{0}^{(2)}, \alpha_{0}^{(2)}, \alpha_{1}^{(2)}\right)$, respectively. We set

and

$$
\left(u, \alpha, \frac{\partial \alpha}{\partial t}\right)=\left(u^{(1)}, \alpha^{(1)}, \frac{\partial \alpha^{(1)}}{\partial t}\right)-\left(u^{(2)}, \alpha^{(2)}, \frac{\partial \alpha^{(2)}}{\partial t}\right)
$$

and have

$$
\left(u_{0}, \alpha_{0}, \alpha_{1}\right)=\left(u_{0}^{(1)}, \alpha_{0}^{(1)}, \alpha_{1}^{(1)}\right)-\left(u_{0}^{(2)}, \alpha_{0}^{(2)}, \alpha_{1}^{(2)}\right)
$$

$$
\begin{gathered}
\frac{\partial u}{\partial t}-\Delta u+f\left(u^{(1)}\right)-f\left(u^{(2)}\right)=\frac{\partial \alpha}{\partial t}-\Delta \frac{\partial \alpha}{\partial t}, \\
\frac{\partial^{2} \alpha}{\partial t^{2}}-\Delta \frac{\partial^{2} \alpha}{\partial t^{2}}-\Delta \frac{\partial \alpha}{\partial t}-\Delta \alpha=-\frac{\partial u}{\partial t} \\
u=\alpha=0 \text { on } \Gamma \\
\left.u\right|_{t=0}=u_{0},\left.\alpha\right|_{t=0}=\alpha_{0},\left.\frac{\partial \alpha}{\partial t}\right|_{t=0}=\alpha_{1} .
\end{gathered}
$$

Multiplying (2.34) by $\frac{\partial u}{\partial t}$, we obtain, owing to (2.33) and the continuous embedding $H^{2}(\Omega) \subset \mathcal{C}(\bar{\Omega})$

$$
\frac{1}{2} \frac{d}{d t}\|\nabla u\|^{2}+\frac{1}{2}\left\|\frac{\partial u}{\partial t}\right\|^{2} \leq\left(\left(\frac{\partial \alpha}{\partial t}-\Delta \frac{\partial \alpha}{\partial t}, \frac{\partial u}{\partial t}\right)\right)+Q\|u\|^{2},
$$

where, here and below,

$$
Q=Q\left(\left\|u_{0}^{(1)}\right\|_{H^{2}(\Omega)},\left\|\alpha_{0}^{(1)}\right\|_{H^{2}(\Omega)},\left\|\alpha_{1}^{(1)}\right\|_{H^{2}(\Omega)},\left\|u_{0}^{(2)}\right\|_{H^{2}(\Omega)},\left\|\alpha_{0}^{(2)}\right\|_{H^{2}(\Omega)},\left\|\alpha_{1}^{(2)}\right\|_{H^{2}(\Omega)}\right)
$$


Multiplying then (2.35) by $\frac{\partial \alpha}{\partial t}-\Delta \frac{\partial \alpha}{\partial t}$ (proceeding exactly as in the previous subsection) and adding the resulting differential equality to (2.38), we find

$$
\frac{d}{d t}\left(\|\nabla u\|^{2}+\|\nabla \alpha\|^{2}+\|\Delta \alpha\|^{2}+\left\|\frac{\partial \alpha}{\partial t}-\Delta \frac{\partial \alpha}{\partial t}\right\|^{2}\right) \leq Q\|u\|^{2} .
$$

It thus follows from (2.39) and Gronwall's lemma that

$$
\begin{gathered}
\|u(t)\|_{H^{1}(\Omega)}^{2}+\|\alpha(t)\|_{H^{2}(\Omega)}^{2}+\left\|\frac{\partial \alpha}{\partial t}(t)\right\|_{H^{2}(\Omega)}^{2} \\
\leq c e^{Q t}\left(\left\|u_{0}\right\|_{H^{1}(\Omega)}^{2}+\left\|\alpha_{0}\right\|_{H^{2}(\Omega)}^{2}+\left\|\alpha_{1}\right\|_{H^{2}(\Omega)}^{2}\right), t \geq 0,
\end{gathered}
$$

hence the uniqueness, as well as the continuity (with respect to the $H^{1}(\Omega) \times H^{2}(\Omega)^{2}$-norm) with respect to the initial data.

It follows from Theorem 2.1 that we can define the family of solving operators

$$
S(t): \Phi \rightarrow \Phi,\left(u_{0}, \alpha_{0}, \alpha_{1}\right) \mapsto\left(u(t), \alpha(t), \frac{\partial \alpha}{\partial t}(t)\right), t \geq 0
$$

where $\Phi=\left(H^{2}(\Omega) \cap H_{0}^{1}(\Omega)\right)^{3}$. Furthermore, this family of solving operators forms a continuous (for the $H^{1}(\Omega) \times H^{2}(\Omega)^{2}$-topology) semigroup, i.e., $S(0)=$ Id and $S(t+\tau)=$ $S(t) \circ S(\tau), \forall t, \tau \geq 0$. Finally, it follows from (2.33) that $S(t)$ is dissipative in $\Phi$, in the sense that it possesses a bounded absorbing set $\mathcal{B}_{0} \subset \Phi$ (i.e., $\forall B \subset \Phi$ bounded, $\exists t_{0}=t_{0}(B)$ such that $t \geq t_{0}$ implies $S(t) B \subset \mathcal{B}_{0}$ ).

Remark 2.2. The dissipativity is a first step in view of the study of the (temporal) asymptotic behavior of the associated dynamical system. In particular, an important issue is to prove the existence of finite-dimensional attractors: such objects describe all possible dynamics of the system; furthermore, the finite-dimensionality means, very roughly speaking, that, even though the initial phase space $\Phi$ has infinite dimension, the reduced dynamics can be described by a finite number of parameters (we refer the interested reader to, e.g., [41] and [44] for discussions on this subject). This will be studied elsewhere.

2.4. Singular nonlinear terms. We now assume that $f$ satisfies

$$
\begin{gathered}
f \in \mathcal{C}^{1}(-1,1), f(0)=0, \\
\lim _{s \rightarrow \pm 1} f(s)= \pm \infty, \lim _{s \rightarrow \pm 1} f^{\prime}(s)=+\infty .
\end{gathered}
$$

In particular, these assumptions are satisfied by the thermodynamically relevant logarithmic nonlinear terms mentioned in the introduction. Furthermore, it follows from these properties that

$$
f^{\prime} \geq-c_{0}, c_{0} \geq 0
$$




$$
f(s) s \geq F(s)-c_{1} \geq-c_{2}, c_{1}, c_{2} \geq 0, s \in(-1,1),
$$

where, again, $F(s)=\int_{0}^{s} f(\xi) d \xi$.

We then introduce, for $N \in \mathbb{N}$ given, the approximated function $f_{N} \in \mathcal{C}^{1}(\mathbb{R})$ given by

$$
f_{N}(s)=\left\{\begin{array}{l}
f\left(-1+\frac{1}{N}\right)+f^{\prime}\left(-1+\frac{1}{N}\right)\left(s+1-\frac{1}{N}\right), s<-1+\frac{1}{N}, \\
f(s),|s| \leq 1-\frac{1}{N}, \\
f\left(1-\frac{1}{N}\right)+f^{\prime}\left(1-\frac{1}{N}\right)\left(s-1+\frac{1}{N}\right), s>1-\frac{1}{N} .
\end{array}\right.
$$

Owing to the properties of $f$ and the above explicit expression of $f_{N}$, it is not difficult to prove that, at least for $N$ large enough,

$$
f_{N}^{\prime} \geq-c_{0}
$$

where $F_{N}(s)=\int_{0}^{s} f_{N}(\xi) d \xi$. Here, all the constants are independent of $N$. The functions $f_{N}$ thus satisfy all the assumptions of the previous subsection (uniformly with respect to $N$ if $N$ is large enough).

We finally consider the following approximated problems:

$$
\begin{gathered}
\frac{\partial u^{N}}{\partial t}-\Delta u^{N}+f_{N}\left(u^{N}\right)=\frac{\partial \alpha^{N}}{\partial t}-\Delta \frac{\partial \alpha^{N}}{\partial t}, \\
\frac{\partial^{2} \alpha^{N}}{\partial t^{2}}-\Delta \frac{\partial^{2} \alpha^{N}}{\partial t^{2}}-\Delta \frac{\partial \alpha^{N}}{\partial t}-\Delta \alpha^{N}=-\frac{\partial u^{N}}{\partial t}, \\
u^{N}=\alpha^{N}=0 \text { on } \Gamma \\
\left.u^{N}\right|_{t=0}=u_{0},\left.\alpha^{N}\right|_{t=0}=\alpha_{0},\left.\frac{\partial \alpha^{N}}{\partial t}\right|_{t=0}=\alpha_{1} .
\end{gathered}
$$

In particular, proceeding as in the previous subsection, we can prove the existence and uniqueness of the solution $\left(u^{N}, \alpha^{N}, \frac{\partial \alpha^{N}}{\partial t}\right)$ to $(2.47)-(2.50)$ and we have the

Theorem 2.3. We assume that (2.41)-(2.42) hold and that $\left(u_{0}, \alpha_{0}, \alpha_{1}\right) \in\left(H^{2}(\Omega) \cap\right.$ $\left.H_{0}^{1}(\Omega)\right)^{3}$, with $\left\|u_{0}\right\|_{L^{\infty}(\Omega)}<1$. Then, (2.47)-(2.50) possesses a unique solution $\left(u, \alpha, \frac{\partial \alpha}{\partial t}\right)$ such that

and

$$
\left(u, \alpha, \frac{\partial \alpha}{\partial t}\right) \in L^{\infty}\left(\mathbb{R}^{+} ; H^{2}(\Omega) \cap H_{0}^{1}(\Omega)\right)^{3}
$$

$$
\frac{\partial u}{\partial t} \in L^{\infty}\left(\mathbb{R}^{+} ; L^{2}(\Omega)\right) \cap L^{2}\left(0, T ; H_{0}^{1}(\Omega)\right), \forall T>0
$$


Furthermore, $-1<u(t, x)<1$ a.e. $(t, x)$.

\section{Proof. a) Existence:}

As mentioned above, (2.47)-(2.50) possesses a unique solution $\left(u^{N}, \alpha^{N}, \frac{\partial \alpha^{N}}{\partial t}\right)$ which enjoys the same regularity as in Theorem 2.1. Furthermore, since the constants in (2.45)(2.46) are independent of $N$, we can derive exactly the same estimates as in Subsection 2.2 on this solution, uniformly with respect to $N$. In particular, this yields that, at least for a subsequence which we do not relabel, there exists a triplet $\left(u, \alpha, \frac{\partial \alpha}{\partial t}\right)$ such that, for every $T>0$,

$$
\begin{gathered}
u^{N} \rightarrow u \text { in } L^{\infty}\left(0, T ; H^{2}(\Omega)\right) \text { weak }-\star \text { and a.e. }(t, x) \in(0, T) \times \Omega, \\
\frac{\partial u^{N}}{\partial t} \rightarrow \frac{\partial u}{\partial t} \text { in } L^{\infty}\left(0, T ; L^{2}(\Omega)\right) \text { weak }-\star \text { and in } L^{2}\left(0, T ; H^{1}(\Omega)\right) \text { weak } \\
\alpha^{N} \rightarrow \alpha \text { in } L^{\infty}\left(0, T ; H^{2}(\Omega)\right) \text { weak }-\star
\end{gathered}
$$

and

$$
\frac{\partial \alpha^{N}}{\partial t} \rightarrow \frac{\partial \alpha}{\partial t} \text { in } L^{\infty}\left(0, T ; H^{2}(\Omega)\right) \text { weak }-\star .
$$

The only difficulty, to pass to the limit in the approximated problems (say, within a proper weak/variational formulation), is to prove that $f_{N}\left(u^{N}\right)$ tends to $f(u)$ in a proper sense. To do so, we need a uniform (with respect to $N$ ) estimate on $f_{N}\left(u^{N}\right)$ in $L^{2}((0, T) \times$ $\Omega$ ). This can be easily obtained by noting that

hence

$$
f_{N}\left(u^{N}\right)=-\frac{\partial u^{N}}{\partial t}+\Delta u^{N}+\frac{\partial \alpha^{N}}{\partial t}-\Delta \frac{\partial \alpha^{N}}{\partial t}
$$

$$
\left\|f_{N}\left(u^{N}\right)\right\| \leq c\left(\left\|u^{N}\right\|_{H^{2}(\Omega)}+\left\|\frac{\partial u^{N}}{\partial t}\right\|+\left\|\frac{\partial \alpha^{N}}{\partial t}\right\|_{H^{2}(\Omega)}\right)
$$

where the constant $c$ is independent of $N$. Actually, we have more and $f_{N}\left(u^{N}\right)$ is bounded in $L^{\infty}\left(0, T ; H^{1}(\Omega)\right)$.

Noting that, a fortiori, $f_{N}\left(u^{N}\right)$ is bounded, uniformly with respect to $N$, in $L^{1}((0, T) \times$ $\Omega$ ), it follows from the explicit expression of $f_{N}$ that

where

$$
\operatorname{meas}\left(E_{N, M}\right) \leq \varphi\left(\frac{1}{N}\right), N \leq M
$$

$$
E_{N, M}=\left\{(t, x) \in(0, T) \times \Omega,\left|u^{M}(t, x)\right|>1-\frac{1}{N}\right\}
$$

and

$$
\varphi(s)=\frac{c}{\max (|f(1-s)|,|f(s-1)|)},
$$


the constant $c$ being independent of $N$ and $M$. We can note indeed that

$$
\int_{0}^{T} \int_{\Omega}\left|f_{M}\left(u^{M}\right)\right| d x d t \geq \int_{E_{N, M}}\left|f_{M}\left(u^{M}\right)\right| d x d t \geq c^{\prime} \operatorname{meas}\left(E_{N, M}\right) \frac{1}{\varphi\left(\frac{1}{N}\right)},
$$

where the constant $c^{\prime}$ is independent of $N$ and $M$. We can pass to the limit $M \rightarrow+\infty$ (employing Fatou's lemma, see (2.51)) and then $N \rightarrow+\infty$ (noting that $\lim _{s \rightarrow 0} \varphi(s)=0$ ) to find

so that

$$
\operatorname{meas}\{(t, x) \in(0, T) \times \Omega,|u(t, x)| \geq 1\}=0
$$

$$
-1<u(t, x)<1 \text { a.e. }(t, x) .
$$

Next, it follows from the above almost everywhere convergence of $u^{N}$ to $u,(2.52)$ and again the explicit expression of $f_{N}$ that

$$
f_{N}\left(u^{N}\right) \rightarrow f(u) \text { a.e. }(t, x) \in(0, T) \times \Omega .
$$

Finally, since $f_{N}\left(u^{N}\right)$ is bounded, uniformly with respect to $N$, in $L^{2}((0, T) \times \Omega)$, it follows from $(2.53)$ that $f_{N}\left(u^{N}\right) \rightarrow f(u)$ in $L^{2}((0, T) \times \Omega)$ weak, which finishes the proof of the passage to the limit.

\section{b) Uniqueness:}

Here, the proof of uniqueness is not as straightforward as in the case of regular nonlinear terms, since, as we cannot expect a bound on $f^{\prime}$, we cannot proceed as in the proof of Theorem 2.1.

Instead, we multiply (2.34) by $u$ and have, owing to (2.43),

$$
\frac{d}{d t}\|u\|^{2}+c\|u\|_{H^{1}(\Omega)}^{2} \leq c\left(\|u\|^{2}+\left\|\frac{\partial \alpha}{\partial t}\right\|_{H^{2}(\Omega)}^{2}\right), c>0 .
$$

Next, we integrate (2.35) between 0 and $t$ to obtain

$$
\frac{\partial \alpha}{\partial t}-\Delta \frac{\partial \alpha}{\partial t}-\Delta \alpha-\Delta \int_{0}^{t} \alpha(\tau) d \tau=-u
$$

Here, we have taken, for simplicity, $\left(u_{0}, \alpha_{0}, \alpha_{1}\right)=(0,0,0)$. Multiplying $(2.55)$ by $-\Delta \alpha$, we find

$$
\frac{d}{d t}\left(\|\nabla \alpha\|^{2}+\|\Delta \alpha\|^{2}+\left\|\Delta \int_{0}^{t} \alpha(\tau) d \tau\right\|^{2}\right)+c\|\alpha\|_{H^{2}(\Omega)}^{2} \leq\|u\|^{2}, c>0 .
$$

Multiplying then $(2.55)$ by $-\Delta \frac{\partial \alpha}{\partial t}$, we have

$$
\frac{d}{d t}\left(\|\Delta \alpha\|^{2}+2\left(\left(\Delta \int_{0}^{t} \alpha(\tau) d \tau, \Delta \alpha\right)\right)\right)+c\left\|\frac{\partial \alpha}{\partial t}\right\|_{H^{2}(\Omega)}^{2} \leq c^{\prime}\left(\|u\|^{2}+\|\alpha\|_{H^{2}(\Omega)}^{2}\right), c>0
$$


Summing finally $\delta_{5}$ times $(2.54),(2.56)$ and $\delta_{6}$ times $(2.57)$, we obtain a differential inequality of the form, taking $\delta_{5}, \delta_{6}>0$ small enough,

$$
\frac{d E_{5}}{d t} \leq c E_{5}
$$

where

$$
\begin{gathered}
E_{5}=\delta_{5}\|u\|^{2}+\|\nabla \alpha\|^{2}+\|\Delta \alpha\|^{2}+\left\|\Delta \int_{0}^{t} \alpha(\tau) d \tau\right\|^{2} \\
+\delta_{6}\left(\|\Delta \alpha\|^{2}+2\left(\left(\Delta \int_{0}^{t} \alpha(\tau) d \tau, \Delta \alpha\right)\right)\right)
\end{gathered}
$$

satisfies

$$
E_{5} \geq c\left(\|u\|^{2}+\|\alpha\|_{H^{2}(\Omega)}^{2}\right), c>0 .
$$

Gronwall's lemma, together with (2.60), then yields the uniqueness. We can note that this would not give a continuity result (with respect to the initial data) for $\frac{\partial \alpha}{\partial t}$, but such a continuity would then follow from (2.55).

Remark 2.4. Since $\left\|u_{0}\right\|_{L^{\infty}(\Omega)}<1$, then, for $N$ large enough, $f_{N}\left(u_{0}\right)=f\left(u_{0}\right)$ and $F_{N}\left(u_{0}\right)=F\left(u_{0}\right)$. This yields

$$
\left|\int_{\Omega} F_{N}\left(u_{0}\right) d x\right| \leq Q\left(\left\|u_{0}\right\|_{H^{2}(\Omega)}\right)
$$

where the monotone increasing function $Q$ is independent of $N$. Now, in the case of the thermodynamically relevant logarithmic nonlinear terms (see the introduction), $F$ is bounded on $(-1,1)$, yielding that also $F_{N}$ is bounded, uniformly with respect to $N$, on $(-1,1)$, since

$$
F_{N}(s)=\left\{\begin{array}{l}
F\left(-1+\frac{1}{N}\right)+f\left(-1+\frac{1}{N}\right)\left(s+1-\frac{1}{N}\right) \\
+\frac{1}{2} f^{\prime}\left(-1+\frac{1}{N}\right)\left(s+1-\frac{1}{N}\right)^{2}, s<-1+\frac{1}{N}, \\
F(s),|s| \leq 1-\frac{1}{N}, \\
F\left(1-\frac{1}{N}\right)+f\left(1-\frac{1}{N}\right)\left(s-1+\frac{1}{N}\right) \\
+\frac{1}{2} f^{\prime}\left(1-\frac{1}{N}\right)\left(s-1+\frac{1}{N}\right)^{2}, s>1-\frac{1}{N} .
\end{array}\right.
$$

In that case, we can relax the assumptions on $u_{0}$ and only assume that $-1<u_{0}(x)<1$ a.e. $x \in \Omega$.

Remark 2.5. We again assume that $\left\|u_{0}\right\|_{L^{\infty}(\Omega)}<1$. We can prove, for the original Caginalp system, a strict separation property of the form

$$
\|u(t)\|_{L^{\infty}(\Omega)} \leq \eta, t \in[0, T], \eta=\eta(T) \in(0,1), T>0 .
$$


Let us assume more regularity on $\alpha_{0}$ and $\alpha_{1}$, namely, $\left(\alpha_{0}, \alpha_{1}\right) \in\left(H^{3}(\Omega) \cap H_{0}^{1}(\Omega)\right)^{2}$. Then, rewriting (2.2) in the (functional) form

$$
\frac{d^{2} \alpha}{d t^{2}}+A \frac{d^{2} \alpha}{d t^{2}}+A \frac{d \alpha}{d t}+A \alpha=-\frac{d u}{d t}
$$

where $A$ denotes the minus Laplace operator with Dirichlet boundary conditions, and multiplying (scalarly) (2.62) by $A^{2} \frac{d \alpha}{d t}$, we have

$$
\frac{d}{d t}\left(\left\|A^{\frac{3}{2}} \alpha\right\|^{2}+\left\|A \frac{d \alpha}{d t}\right\|^{2}+\left\|A^{\frac{3}{2}} \frac{d \alpha}{d t}\right\|^{2}\right)+\left\|A^{\frac{3}{2}} \frac{d \alpha}{d t}\right\|^{2} \leq\left\|A^{\frac{1}{2}} \frac{d u}{d t}\right\|^{2},
$$

which yields that $\frac{\partial \alpha}{\partial t}-\Delta \frac{\partial \alpha}{\partial t} \in L^{\infty}\left(0, T ; H^{1}(\Omega)\right)$. Having this, we can deduce a strict separation property as above, in one and two space dimensions. Indeed, it has been proved in [31] that, in one and two space dimensions, the (regular) solution to the parabolic equation

$$
\frac{\partial u}{\partial t}-\Delta u+f(u)=g, g=g(t, x) \in L^{\infty}\left(0, T ; H^{1}(\Omega)\right),
$$

satisfies (2.61). Actually, in one space dimension, this is straightforward, owing to the maximum/comparison principle and the continuous embedding $H^{1}(\Omega) \subset \mathcal{C}(\bar{\Omega})$. In two space dimensions, the proof is more involved and is based on a proper Orlicz embedding (see also [40]). Finally, in three space dimensions, (2.61) also holds, but provided that the singular function $|f|$ has a growth of the form

$$
\frac{c}{\left(1-s^{2}\right)^{r}}, r \geq 5, c>0
$$

close to \pm 1 . Unfortunately, this is not satisfied by the thermodynamically relevant logarithmic nonlinear terms and, in that case, the strict separation from the pure phases is an open problem.

\section{NEUMANN BOUNDARY CONDITIONS}

We now consider the following initial and boundary value problem:

$$
\begin{gathered}
\frac{\partial u}{\partial t}-\Delta u+f(u)=\frac{\partial \alpha}{\partial t}-\Delta \frac{\partial \alpha}{\partial t} \\
\frac{\partial^{2} \alpha}{\partial t^{2}}-\Delta \frac{\partial^{2} \alpha}{\partial t^{2}}-\Delta \frac{\partial \alpha}{\partial t}-\Delta \alpha=-\frac{\partial u}{\partial t} \\
\frac{\partial u}{\partial \nu}=\frac{\partial \alpha}{\partial \nu}=0 \text { on } \Gamma, \\
\left.u\right|_{t=0}=u_{0},\left.\alpha\right|_{t=0}=\alpha_{0},\left.\frac{\partial \alpha}{\partial t}\right|_{t=0}=\alpha_{1},
\end{gathered}
$$


where $\nu$ denotes the unit outer normal, and we assume that $f$ satisfies (2.5)-(2.7) (note however that the assumption $f(0)=0$ is not necessary in that case).

3.1. A priori estimates. We first note that, integrating (3.2) over $\Omega$, we have, in view of $(3.3)$,

$$
\frac{d^{2}\langle\alpha\rangle}{d t^{2}}=-\frac{d\langle u\rangle}{d t}
$$

It thus follows from (3.4) and (3.5) that

$$
\frac{d\langle\alpha\rangle}{d t}=\left\langle u_{0}+\alpha_{1}\right\rangle-\langle u\rangle
$$

meaning, in particular, that $\left\langle u+\frac{\partial \alpha}{\partial t}\right\rangle$ is a conserved quantity.

This constitutes the main difference, when compared with Dirichlet boundary conditions, and obliges us to be more careful in the derivation of the a priori estimates. In particular, in order to still have dissipativity, we assume from now on that

$$
\left|\left\langle u_{0}+\alpha_{1}\right\rangle\right| \leq M, M \geq 0 \text { given, }
$$

hence

$$
\left|\left\langle u+\frac{\partial \alpha}{\partial t}\right\rangle(t)\right| \leq M, t \geq 0
$$

We deduce from (3.2) and (3.5) that

$$
\frac{\partial^{2} \bar{\alpha}}{\partial t^{2}}-\Delta \frac{\partial^{2} \bar{\alpha}}{\partial t^{2}}-\Delta \frac{\partial \bar{\alpha}}{\partial t}-\Delta \bar{\alpha}=-\frac{\partial \bar{u}}{\partial t} .
$$

Furthermore, we can rewrite, in view of (3.6), (3.1) as

$$
\frac{\partial u}{\partial t}-\Delta u+f(u)=\frac{\partial \bar{\alpha}}{\partial t}-\Delta \frac{\partial \bar{\alpha}}{\partial t}+\left\langle u_{0}+\alpha_{1}\right\rangle-\langle u\rangle .
$$

Multiplying (3.10) by $\frac{\partial u}{\partial t}$, we have

$$
\begin{gathered}
\frac{1}{2} \frac{d}{d t}\left(\|\nabla u\|^{2}+2 \int_{\Omega} F(u) d x\right)+\left\|\frac{\partial u}{\partial t}\right\|^{2} \\
=\left(\left(\frac{\partial \bar{\alpha}}{\partial t}-\Delta \frac{\partial \bar{\alpha}}{\partial t}, \frac{\partial \bar{u}}{\partial t}\right)\right)-\operatorname{Vol}(\Omega)\langle u\rangle\left\langle\frac{\partial u}{\partial t}\right\rangle+\operatorname{Vol}(\Omega)\left\langle u_{0}+\alpha_{1}\right\rangle\left\langle\frac{\partial u}{\partial t}\right\rangle,
\end{gathered}
$$

which yields, owing to (3.7),

$$
\frac{d}{d t}\left(\|\nabla u\|^{2}+\operatorname{Vol}(\Omega)\langle u\rangle^{2}+2 \int_{\Omega} F(u) d x\right)+\left\|\frac{\partial u}{\partial t}\right\|^{2} \leq 2\left(\left(\frac{\partial \bar{\alpha}}{\partial t}-\Delta \frac{\partial \bar{\alpha}}{\partial t}, \frac{\partial \bar{u}}{\partial t}\right)\right)+c_{M} .
$$

We then multiply $(3.9)$ by $\frac{\partial \bar{\alpha}}{\partial t}-\Delta \frac{\partial \bar{\alpha}}{\partial t}$ to obtain 


$$
\begin{aligned}
\frac{d}{d t}\left(\|\nabla \bar{\alpha}\|^{2}+\|\Delta \bar{\alpha}\|^{2}\right. & \left.+\left\|\frac{\partial \bar{\alpha}}{\partial t}-\Delta \frac{\partial \bar{\alpha}}{\partial t}\right\|^{2}\right)+2\left\|\nabla \frac{\partial \bar{\alpha}}{\partial t}\right\|^{2}+2\left\|\Delta \frac{\partial \bar{\alpha}}{\partial t}\right\|^{2} \\
& =-2\left(\left(\frac{\partial \bar{\alpha}}{\partial t}-\Delta \frac{\partial \bar{\alpha}}{\partial t}, \frac{\partial \bar{u}}{\partial t}\right)\right) .
\end{aligned}
$$

Summing finally (3.11) and (3.12), we find

$$
\begin{gathered}
\frac{d}{d t}\left(\|\nabla u\|^{2}+\operatorname{Vol}(\Omega)\langle u\rangle^{2}+2 \int_{\Omega} F(u) d x+\|\nabla \bar{\alpha}\|^{2}+\|\Delta \bar{\alpha}\|^{2}+\left\|\frac{\partial \bar{\alpha}}{\partial t}-\Delta \frac{\partial \bar{\alpha}}{\partial t}\right\|^{2}\right) \\
+c\left(\left\|\frac{\partial u}{\partial t}\right\|^{2}+\left\|\frac{\partial \bar{\alpha}}{\partial t}\right\|_{H^{2}(\Omega)}^{2}\right) \leq c_{M}^{\prime}, \quad c>0 .
\end{gathered}
$$

Next, multiplying (3.10) by $u$, we have, proceeding as in Subsection 2.2,

$$
\frac{d}{d t}\|u\|^{2}+c\left(\|\nabla u\|^{2}+\operatorname{Vol}(\Omega)\langle u\rangle^{2}+2 \int_{\Omega} F(u) d x\right) \leq c^{\prime}\left\|\frac{\partial \bar{\alpha}}{\partial t}\right\|_{H^{2}(\Omega)}^{2}+c_{M}^{\prime \prime}, c>0 .
$$

We now note that, owing to (3.6),

$$
\left\langle\frac{\partial \alpha}{\partial t}\right\rangle^{2} \leq 2\left(\langle u\rangle^{2}+M^{2}\right)
$$

and, owing to (3.5)-(3.6),

$$
\begin{gathered}
\frac{d}{d t}\left\langle\frac{\partial \alpha}{\partial t}\right\rangle^{2}=2\left\langle\frac{\partial^{2} \alpha}{\partial t^{2}}\right\rangle\left(\left\langle u_{0}+\alpha_{1}\right\rangle-\langle u\rangle\right) \\
\leq c\left(\langle u\rangle^{2}+\left\langle\frac{\partial u}{\partial t}\right\rangle^{2}+M^{2}\right) .
\end{gathered}
$$

Summing (3.13), $\delta_{7}$ times (3.14), $\delta_{8}$ times (3.15) and $\delta_{9}$ times (3.16), where $\delta_{7}, \delta_{8}, \delta_{9}>0$ are small enough, we obtain a differential inequality of the form

$$
\frac{d E_{6}}{d t}+c\left(\|u\|_{H^{1}(\Omega)}^{2}+2 \int_{\Omega} F(u) d x+\left\|\frac{\partial u}{\partial t}\right\|^{2}+\left\|\frac{\partial \alpha}{\partial t}\right\|_{H^{2}(\Omega)}^{2}\right) \leq c_{M}^{\prime}, c>0
$$

where $E_{6}$ satisfies

$$
E_{6} \geq c\left(\|u\|_{H^{1}(\Omega)}^{2}+\int_{\Omega} F(u) d x+\|\bar{\alpha}\|_{H^{2}(\Omega)}^{2}+\left\|\frac{\partial \alpha}{\partial t}\right\|_{H^{2}(\Omega)}^{2}\right)-c^{\prime}, c>0 .
$$

Having this, we can proceed as in Subsection 2.2, with minor modifications, and derive a differential inequality of the form

$$
\frac{d E_{7}}{d t}+c\left(E_{7}+\|u\|_{H^{2}(\Omega)}^{2}+\left\|\frac{\partial u}{\partial t}\right\|_{H^{1}(\Omega)}^{2}\right) \leq c_{M}^{\prime}, c>0
$$

where $E_{7}$ satisfies 


$$
E_{7} \geq c\left(\|u\|_{H^{1}(\Omega)}^{2}+\int_{\Omega} F(u) d x+\left\|\frac{\partial u}{\partial t}\right\|^{2}+\|\bar{\alpha}\|_{H^{2}(\Omega)}^{2}+\left\|\frac{\partial \alpha}{\partial t}\right\|_{H^{2}(\Omega)}^{2}\right)-c^{\prime}, c>0,
$$

together with

$$
\|u(t)\|_{H^{2}(\Omega)}^{2} \leq c E_{7}(t)+c_{M}^{\prime}, t \geq 0
$$

Remark 3.1. Here, we are not able to derive a dissipative estimate on $\langle\alpha\rangle$ and, thus, on $\alpha$. Indeed, the best that we have is, in view of (3.6),

$$
|\langle\alpha(t)\rangle| \leq M t+\int_{0}^{t}|\langle u(\xi)\rangle| d \xi,
$$

which is not dissipative. However, $\bar{\alpha}=\alpha-\langle\alpha\rangle$ is dissipative.

\subsection{The dissipative semigroup. We have the}

Theorem 3.2. We assume that (2.5)-(2.7) hold. Then, for every $\left(u_{0}, \alpha_{0}, \alpha_{1}\right) \in H^{2}(\Omega)^{3}$ such that $\frac{\partial u_{0}}{\partial \nu}=\frac{\partial \alpha_{0}}{\partial \nu}=\frac{\partial \alpha_{1}}{\partial \nu}=0$ on $\Gamma,(3.1)-(3.4)$ possesses a unique solution $\left(u, \alpha, \frac{\partial \alpha}{\partial t}\right)$ such that

$$
\begin{gathered}
\left(u, \bar{\alpha}, \frac{\partial \alpha}{\partial t}\right) \in L^{\infty}\left(\mathbb{R}^{+} ; H^{2}(\Omega)\right)^{3}, \\
\alpha \in L^{\infty}\left(0, T ; H^{2}(\Omega)\right)
\end{gathered}
$$

and

$\forall T>0$.

$$
\frac{\partial u}{\partial t} \in L^{\infty}\left(\mathbb{R}^{+} ; L^{2}(\Omega)\right) \cap L^{2}\left(0, T ; H^{1}(\Omega)\right)
$$

The proof of this theorem is similar to that of Theorem 2.1, with minor modifications as far as the proof of uniqueness is concerned. Now, concerning the proof of the continuous dependence with respect to the initial data, we again proceed in a same way, assuming, for simplicity, that $\left\langle u_{0}^{(1)}+\alpha_{0}^{(1)}\right\rangle=\left\langle u_{0}^{(2)}+\alpha_{0}^{(2)}\right\rangle$.

Setting then

$$
\Phi_{M}=\left\{(v, p, q) \in H^{2}(\Omega)^{2}, \frac{\partial v}{\partial \nu}=\frac{\partial p}{\partial \nu}=\frac{\partial q}{\partial \nu}=0 \text { on } \Gamma,\langle v+q\rangle=M\right\}, M \geq 0 \text { given, }
$$

we can define the family of solving operators

$$
S(t): \Phi_{M} \rightarrow \Phi_{M}, \quad\left(u_{0}, \alpha_{0}, \alpha_{1}\right) \mapsto\left(u(t), \alpha(t), \frac{\partial \alpha}{\partial t}(t)\right), t \geq 0 .
$$

This family of operators forms a continuous (for the $H^{1}(\Omega) \times H^{2}(\Omega)^{2}$-topology) semigroup which is not dissipative. However, setting now

$$
\bar{\Phi}_{M}=\left\{(v, p, q) \in \Phi_{M},\langle p\rangle=0\right\}
$$


and defining

$$
\bar{S}(t): \bar{\Phi}_{M} \rightarrow \bar{\Phi}_{M},\left(u_{0}, \bar{\alpha}_{0}, \alpha_{1}\right) \mapsto\left(u(t), \bar{\alpha}(t), \frac{\partial \alpha}{\partial t}(t)\right), t \geq 0,
$$

we still have a semigroup (see [12]) and it follows from (3.19)-(3.20) that this semigroup is dissipative in $\bar{\Phi}_{M}$.

We can also easily adapt the estimates in Subsection 3.1 (and also in Subsection 2.4) to singular nonlinear terms (again, with minor changes) and we have the

Theorem 3.3. We assume that (2.41)-(2.42) hold. Then, for every $\left(u_{0}, \alpha_{0}, \alpha_{1}\right) \in H^{2}(\Omega)^{3}$ such that $\frac{\partial u_{0}}{\partial \nu}=\frac{\partial \alpha_{0}}{\partial \nu}=\frac{\partial \alpha_{1}}{\partial \nu}=0$ on $\Gamma$ and $\left\|u_{0}\right\|_{L^{\infty}(\Omega)}<1$, (3.1)-(3.4) possesses a unique solution $\left(u, \alpha, \frac{\partial \alpha}{\partial t}\right)$ such that

$$
\begin{gathered}
\left(u, \bar{\alpha}, \frac{\partial \alpha}{\partial t}\right) \in L^{\infty}\left(\mathbb{R}^{+} ; H^{2}(\Omega)\right)^{3}, \\
\alpha \in L^{\infty}\left(0, T ; H^{2}(\Omega)\right)
\end{gathered}
$$

and

$$
\frac{\partial u}{\partial t} \in L^{\infty}\left(\mathbb{R}^{+} ; L^{2}(\Omega)\right) \cap L^{2}\left(0, T ; H^{1}(\Omega)\right),
$$

$\forall T>0$. Furthermore, $-1<u(t, x)<1$ a.e. $(t, x)$.

\section{Spatial BeHAVIOR OF SOlUtions in A SEMI-INFINITE CYLINDER}

4.1. Phragmén-Lindelöf alternative. Our aim in this subsection is to study the spatial behavior of the solutions to (2.1)-(2.2). More precisely, we study this system in the semiinfinite cylinder $R=(0,+\infty) \times D$, where $D$ is a two-dimensional bounded domain which is smooth enough to allow the use of the divergence theorem. We supplement the equations with the following boundary conditions:

$$
\begin{gathered}
u=\alpha=0 \text { on }(0, T) \times(0,+\infty) \times \partial D, \\
u\left(t, 0, x_{2}, x_{3}\right)=h\left(t, x_{2}, x_{3}\right), \alpha\left(t, 0, x_{2}, x_{3}\right)=m\left(t, x_{2}, x_{3}\right) \text { on }(0, T) \times\{0\} \times D,
\end{gathered}
$$

where $T>0$ is a given final time, and null initial conditions:

$$
\left.u\right|_{t=0}=\left.\alpha\right|_{t=0}=\left.\frac{\partial \alpha}{\partial t}\right|_{t=0}=0 \text { on } R .
$$

The nonlinearity of the problem is determined by the function $f$. As far as this function is concerned, we assume that there exists a positive constant $d$ such that

$$
f(s) s+d s^{2} \geq 0 \text { and } F(s)+d s^{2} \geq 0 .
$$

Here, $F$ is as defined in the previous sections. It is clear that the function $f(s)=s^{3}-s$ satisfies these conditions. Actually, any function of the form $f(s)=a|s|^{k} s-b s, a, k>0$, satisfies the assumptions.

Our aim is to obtain an alternative of Phragmén-Lindelöf type, i.e., the solutions decrease (resp., grow) in a negative (resp., positive) exponential way with respect to the spatial variable. 
We start our analysis by considering the function

$$
F_{\omega}(t, z)=\int_{0}^{t} \int_{D(z)} \exp (-2 \omega s)\left(u_{, 1} u_{s}+\alpha_{s}\left(\alpha_{s, 1}+\alpha_{, 1}\right)\right) d a d s
$$

where $D(z)=\left\{x \in R, x_{1}=z\right\}$ and $\omega$ is an arbitrary positive constant to be fixed later; here, $v_{s}=\frac{\partial v}{\partial s}$ and $v_{, 1}=\frac{\partial v}{\partial x_{1}}$. We have, owing to the boundary and initial conditions and the divergence theorem,

$$
F_{\omega}(t, z+h)-F_{\omega}(t, z)=\int_{0}^{t} \int_{R(z, z+h)} \exp (-2 \omega s) W d x d s
$$

where $R(z, z+h)=\left\{x \in R, z<x_{1}<z+h\right\}$ and

$$
W=u_{, i} u_{s, i}+u_{s}^{2}+f(u) u_{s}-\left(\alpha_{s}-\triangle \alpha_{s}\right) u_{s}+\alpha_{s, i} \alpha_{s, i}+\alpha_{, i} \alpha_{s, i}+\left(\triangle \alpha_{s}+\triangle \alpha\right) \alpha_{s} .
$$

We thus see that

$W=\frac{d}{d s}\left(\frac{1}{2}|\nabla u|^{2}+F(u)+\frac{1}{2}\left(\alpha_{s}-\triangle \alpha_{s}\right)^{2}+\frac{1}{2}|\nabla \alpha|^{2}+\frac{1}{2}(\triangle \alpha)^{2}\right)+\left|u_{s}\right|^{2}+\left(\triangle \alpha_{s}\right)^{2}+\left|\nabla \alpha_{s}\right|^{2}$.

This yields

$$
\begin{aligned}
& F_{\omega}(t, z+h)-F_{\omega}(t, z) \\
& =\frac{\exp (-2 \omega t)}{2} \int_{R(z, z+h)}\left(|\nabla u|^{2}+2 F(u)+\left(\alpha_{s}-\triangle \alpha_{s}\right)^{2}+|\nabla \alpha|^{2}+(\triangle \alpha)^{2}\right) d x \\
& \quad+\int_{0}^{t} \int_{R(z, z+h)} \exp (-2 \omega s)\left(\left|u_{s}\right|^{2}+\left(\triangle \alpha_{s}\right)^{2}+\left|\nabla \alpha_{s}\right|^{2}\right. \\
& \left.\quad+\omega\left(|\nabla u|^{2}+2 F(u)+\left(\alpha_{s}-\triangle \alpha_{s}\right)^{2}+|\nabla \alpha|^{2}+(\triangle \alpha)^{2}\right)\right) d x d s
\end{aligned}
$$

and a direct differentiation gives

$$
\begin{gathered}
\frac{\partial F_{\omega}(t, z)}{\partial z}=\frac{\exp (-2 \omega t)}{2} \int_{D(z)}\left(|\nabla u|^{2}+2 F(u)+\left(\alpha_{s}-\triangle \alpha_{s}\right)^{2}+|\nabla \alpha|^{2}+(\triangle \alpha)^{2}\right) d a \\
\quad+\int_{0}^{t} \int_{D(z)} \exp (-2 \omega s)\left(\left|u_{s}\right|^{2}+\left(\triangle \alpha_{s}\right)^{2}+\left|\nabla \alpha_{s}\right|^{2}\right. \\
\left.\quad+\omega\left(|\nabla u|^{2}+2 F(u)+\left(\alpha_{s}-\triangle \alpha_{s}\right)^{2}+|\nabla \alpha|^{2}+(\triangle \alpha)^{2}\right)\right) d a d s
\end{gathered}
$$

We now consider a second functional, namely,

$$
G_{\omega}(t, z)=\int_{0}^{t} \int_{D(z)} \exp (-2 \omega s) u_{, 1} u d a d s .
$$

We find, proceeding as above,

$$
\begin{aligned}
G_{\omega}(t, z+ & h)-G_{\omega}(t, z)=\frac{\exp (-2 \omega t)}{2} \int_{R(z, z+h)}|u|^{2} d x \\
& +\int_{0}^{t} \int_{R(z, z+h)} \exp (-2 \omega s)\left(|\nabla u|^{2}+f(u) u-\left(\alpha_{s}-\triangle \alpha_{s}\right) u+\omega|u|^{2}\right) d x d s
\end{aligned}
$$


and we see that

$$
\begin{aligned}
\frac{\partial G_{\omega}(t, z)}{\partial z} & =\frac{\exp (-2 \omega t)}{2} \int_{D(z)}|u|^{2} d a \\
& +\int_{0}^{t} \int_{D(z)} \exp (-2 \omega s)\left(|\nabla u|^{2}+f(u) u-\left(\alpha_{s}-\triangle \alpha_{s}\right) u+\omega|u|^{2}\right) d a d s
\end{aligned}
$$

Next, we consider a positive constant $\phi$ to be fixed later and introduce the function $H_{\omega}=F_{\omega}+\phi G_{\omega}$. We have

$$
\begin{aligned}
\frac{\partial H_{\omega}(t, z)}{\partial z}=\frac{\exp (-2 \omega t)}{2} \int_{D(z)} & \left(|\nabla u|^{2}+2 F(u)+\left(\alpha_{s}-\triangle \alpha_{s}\right)^{2}+|\nabla \alpha|^{2}\right. \\
& \left.+(\triangle \alpha)^{2}+\phi|u|^{2}\right) d x+\int_{0}^{t} \int_{D(z)} \exp (-2 \omega s) \Sigma_{\phi} d x d s
\end{aligned}
$$

where

$$
\begin{aligned}
\Sigma_{\phi}=\left|u_{s}\right|^{2}+\left(\triangle \alpha_{s}\right)^{2}+\left|\nabla \alpha_{s}\right|^{2}+\phi\left(|\nabla u|^{2}+f(u) u-\left(\alpha_{s}-\triangle \alpha_{s}\right) u\right) \\
+\omega\left(|\nabla u|^{2}+2 F(u)+\left(\alpha_{s}-\triangle \alpha_{s}\right)^{2}+|\nabla \alpha|^{2}+(\triangle \alpha)^{2}+\phi|u|^{2}\right) .
\end{aligned}
$$

We can select a positive constant $\phi$ which is large enough to guarantee that

$$
\phi|u|^{2}+2 F(u) \geq 0
$$

and such that

$$
\phi f(u) u+\omega\left(\alpha_{s}-\triangle \alpha_{s}\right)^{2}-\phi\left(\alpha_{s}-\triangle \alpha_{s}\right) u+\phi \omega|u|^{2}+2 \omega F(u) \geq C_{0}\left(\left(\alpha_{s}-\triangle \alpha_{s}\right)^{2}+u^{2}\right),
$$

where $C_{0}$ is a positive constant.

It is worth giving an explanation on why we can choose $\phi$ and $\omega$ satisfying these conditions. The first one is clear and, as far as the second one is concerned, we can note that the determinant of the matrix

$$
\left(\begin{array}{cc}
\phi \omega-2 \omega d-\phi d & \frac{\phi}{2} \\
\frac{\phi}{2} & \omega
\end{array}\right)
$$

reads $\phi \omega^{2}-2 \omega^{2} d-\phi \omega d-\phi^{2} / 4$. We can thus select $\omega$ and $\phi$ such that the above matrix is positive definite and both conditions are satisfied. We thus have the existence of a positive constant $C_{1}$ such that

$\Sigma_{\phi} \geq C_{1}\left(\left|u_{s}\right|^{2}+\left(\triangle \alpha_{s}\right)^{2}+\left|\nabla \alpha_{s}\right|^{2}+|\nabla u|^{2}+\left(\alpha_{s}-\triangle \alpha_{s}\right)^{2}+|\nabla \alpha|^{2}+(\triangle \alpha)^{2}+|u|^{2}+\left(F(u)+d u^{2}\right)\right)$.

The next step consists in obtaining an estimate on $\left|H_{\omega}\right|$ in terms of the spatial derivative of $H_{\omega}$. We note that we can find positive constants $C_{2}, C_{3}$ and $C_{4}$ such that

$$
\left|u_{, 1} u_{s}\right| \leq C_{2} \Sigma_{\phi},\left|\alpha_{s}\left(\alpha_{s, 1}+\alpha_{, 1}\right)\right| \leq C_{3} \Sigma_{\phi} \text { and }\left|\phi u_{, 1} u\right| \leq C_{4} \Sigma_{\phi} .
$$

It then follows that there exists a positive constant $C_{5}=C_{2}+C_{3}+C_{4}$ such that

$$
\left|H_{\omega}(t, z)\right| \leq C_{5} \frac{\partial H_{\omega}(t, z)}{\partial z},
$$

for every $t$ and $z \geq 0$. 
Inequality (4.14) is classical in the study of spatial estimates (see [16]) and yields a Phragmén-Lindelöf alternative. If there exists $z_{0} \geq 0$ such that $H_{\omega}\left(t, z_{0}\right)>0$, then the solution satisfies the estimate

$$
H_{\omega}(t, z) \geq H_{\omega}\left(t, z_{0}\right) \exp \left(C_{5}^{-1}\left(z-z_{0}\right)\right), z \geq z_{0} .
$$

This estimate gives information in terms of the measure defined in the cylinder. Indeed, it follows that

$$
\begin{aligned}
\frac{\exp (-2 \omega t)}{2} \int_{R(0, z)}\left(|\nabla u|^{2}+2 F(u)+\left(\alpha_{s}-\Delta \alpha_{s}\right)^{2}\right. & \left.+|\nabla \alpha|^{2}+(\triangle \alpha)^{2}+\phi|u|^{2}\right) d x \\
& +\int_{0}^{t} \int_{R(0, z)} \exp (-2 \omega s) \Sigma_{\phi} d x d s
\end{aligned}
$$

tends to infinity exponentially fast, where $R(0, z)=\left\{x \in R, x_{1} \leq z\right\}$. On the contrary, when $H_{\omega}(t, z) \leq 0$, for every $z \geq 0$, we deduce that the solution decays and we can obtain an estimate of the form

$$
-H_{\omega}(t, z) \leq-H_{\omega}(t, 0) \exp \left(-C_{5}^{-1} z\right), \quad z \geq 0 .
$$

This inequality implies that $H_{\omega}(t, z)$ tends to zero as $z$ goes to infinity. Furthermore, in view of (4.17), we see that

$$
E_{\omega}(t, z) \leq E_{\omega}(t, 0) \exp \left(-C_{5}^{-1} z\right), z \geq 0
$$

where

$$
\begin{aligned}
& E_{\omega}(t, z) \\
= & \frac{\exp (-2 \omega t)}{2} \int_{R(z)}\left(|\nabla u|^{2}+2 F(u)+\left(\alpha_{s}-\triangle \alpha_{s}\right)^{2}\right. \\
& \left.+|\nabla \alpha|^{2}+(\triangle \alpha)^{2}+\phi|u|^{2}\right) d x \\
& +\int_{0}^{t} \int_{R(z)} \exp (-2 \omega s) \Sigma_{\phi} d x d s
\end{aligned}
$$

and $R(z)=\left\{x \in R, x_{1}>z\right\}$.

Setting finally

$$
\begin{aligned}
\mathcal{E}(t, z)=\frac{1}{2} \int_{R(z)}\left(|\nabla u|^{2}+2 F(u)+\left(\alpha_{s}-\triangle \alpha_{s}\right)^{2}+|\nabla \alpha|^{2}+\right. & \left.(\triangle \alpha)^{2}+\phi|u|^{2}\right) d x \\
& +\int_{0}^{t} \int_{R(z)} \Sigma_{\phi} d x d s
\end{aligned}
$$

we have the

Theorem 4.1. Let $(u, \alpha)$ be a smooth solution to the problem defined by (2.1)-(2.2), the boundary conditions (4.1)-(4.2) and the initial conditions (4.3). Then, either this solution satisfies the growth estimate (4.15) or it satisfies the decay estimate

$$
\mathcal{E}(t, z) \leq E_{\omega}(t, 0) \exp \left(2 \omega t-C_{5}^{-1} z\right), z \geq 0,
$$

where the energy $\mathcal{E}_{\omega}$ is defined in (4.20) and $E_{\omega}$ is given by (4.19). 
4.2. The amplitude term. The spatial decay estimate obtained in the previous subsection is of limited use unless we have an upper bound on the amplitude term $E_{\omega}(t, 0)$ in terms of the boundary conditions. The aim of this subsection is thus to obtain such a bound. To do so, we need to impose an extra condition on the nonlinear term. More precisely, we assume from now on that there exists a positive constant $d_{1}$ such that the inequality

$$
\left(f(s)+d_{1} s\right)^{p} \leq m\left(F(s)+d s^{2}\right), \quad p>1, m \geq 0,
$$

holds. We can note that the functions of the form $f(s)=a|s|^{k} s-d_{1} s, a, k>0$, satisfy this condition.

We first note that

$$
E_{\omega}(t, 0)=-\int_{0}^{t} \int_{D} \exp (-2 \omega s)\left(u_{, 1}\left(g_{s}+\phi g\right)+\left(\alpha_{s, 1}+\alpha_{, 1}\right) l_{s}\right) d a d s
$$

whenever $g$ and $l$ satisfy the same boundary conditions as $u$ and $\alpha$, respectively.

We now define the functions

$$
g(s, x)=h\left(s, x_{2}, x_{3}\right) \exp \left(-b x_{1}\right), \quad l(s, x)=m\left(s, x_{2}, x_{3}\right) \exp \left(-b x_{1}\right),
$$

where $b$ is an arbitrary positive constant. Then,

$$
\begin{gathered}
E_{\omega}(t, 0)=\int_{0}^{t} \int_{R} \exp (-2 \omega s)\left(u_{, i}\left(g_{s, i}+\phi g_{, i}\right)+\left(\alpha_{s, i}+\alpha_{, i}\right) l_{s, i}\right) d x d s \\
\quad+\int_{0}^{t} \int_{R} \exp (-2 \omega s)\left(\left(u_{s}+\left(f(u)+d_{1} u\right)-d_{1} u\right.\right. \\
\left.\left.\quad-\left(\alpha_{s}-\triangle \alpha_{s}\right)\right)\left(g_{s}+\phi g\right)-\left(\alpha_{s}-\triangle \alpha_{s}\right) l_{s s}\right) d x d s \\
+\int_{0}^{t} \int_{R} \exp (-2 \omega s)\left(2 \omega\left(\alpha_{s}-\triangle \alpha_{s}\right)-u_{s}\right) l_{s} d x d s+\exp (-2 \omega t) \int_{R}\left(\alpha_{t}-\triangle \alpha_{t}\right) l_{t} d x
\end{gathered}
$$

Furthermore,

$$
\begin{gathered}
\int_{0}^{t} \int_{R} \exp (-2 \omega s) u_{, i}\left(g_{s, i}+\phi g_{, i}\right) d x d s \leq \epsilon_{1} E_{\omega}(t, 0) \\
+C_{1}^{*} \int_{0}^{t} \int_{R} \exp (-2 \omega s)\left(g_{s, i}+\phi g_{, i}\right)\left(g_{s, i}+\phi g_{, i}\right) d x d s \\
\int_{0}^{t} \int_{R} \exp (-2 \omega s)\left(\alpha_{s, i}+\alpha_{, i}\right) l_{s, i} d x d s \leq \epsilon_{2} E_{\omega}(t, 0)+C_{2}^{*} \int_{0}^{t} \int_{R} \exp (-2 \omega s) l_{s, i} l_{s, i} d x d s, \\
\int_{0}^{t} \int_{R} \exp (-2 \omega s)\left(u_{s}-d_{1} u\right)\left(g_{s}+\phi g\right) d x d s \\
\leq \epsilon_{3} E_{\omega}(t, 0)+C_{3}^{*} \int_{0}^{t} \int_{R} \exp (-2 \omega s)\left(g_{s}+\phi g\right)^{2} d x d s, \\
\int_{0}^{t} \int_{R} \exp (-2 \omega s)\left(f(u)+d_{1} u\right)\left(g_{s}+\phi g\right) d x d s \\
\leq\left(\int_{0}^{t} \int_{R} \exp (-2 \omega s)\left(f(u)+d_{1} u\right)^{p} d x d s\right)^{1 / p}\left(\int_{0}^{t} \int_{R} \exp (-2 \omega s)\left(g_{s}+\phi g\right)^{q} d x d s\right)^{1 / q}
\end{gathered}
$$




$$
\begin{gathered}
\leq\left(\int_{0}^{t} \int_{R} \exp (-2 \omega s) m\left(F(u)+d u^{2}\right) d x d s\right)^{1 / p}\left(\int_{0}^{t} \int_{R} \exp (-2 \omega s)\left(g_{s}+\phi g\right)^{q} d x d s\right)^{1 / q} \\
\leq \epsilon_{4} E_{\omega}(t, 0)+C_{4}^{*} \int_{0}^{t} \int_{R} \exp (-2 \omega s)\left(g_{s}+\phi g\right)^{q} d x d s \\
-\int_{0}^{t} \int_{R} \exp (-2 \omega s)\left(\alpha_{s}-\triangle \alpha_{s}\right)\left(g_{s}+\phi g\right) d x d s \leq \epsilon_{5} E_{\omega}(t, 0) \\
+C_{5}^{*} \int_{0}^{t} \int_{R} \exp (-2 \omega s)\left(g_{s}+\phi g\right)^{2} d x d s \\
-\int_{0}^{t} \int_{R} \exp (-2 \omega s)\left(\alpha_{s}-\triangle \alpha_{s}\right) l_{s s} d x d s \leq \epsilon_{6} E_{\omega}(t, 0)+C_{6}^{*} \int_{0}^{t} \int_{R} \exp (-2 \omega s) l_{s s}^{2} d x d s, \\
\int_{0}^{t} \int_{R} \exp (-2 \omega s)\left(2 \omega\left(\alpha_{s}-\triangle \alpha_{s}\right)-u_{s}\right) l_{s} d x d s \leq \epsilon_{7} E_{\omega}(t, 0)+C_{7}^{*} \int_{0}^{t} \int_{R} \exp (-2 \omega s) l_{s}^{2} d x d s \\
\exp (-2 \omega t) \int_{R}\left(\alpha_{s}-\triangle \alpha_{s}\right) l_{s} d x \leq \epsilon_{8} E_{\omega}(t, 0)+C_{8}^{*} \int_{R} \exp (-2 \omega t) l_{t}^{2} d x .
\end{gathered}
$$

Here, $\epsilon_{i}, i=1, \ldots, 8$, are positive constants to be fixed later and $C_{i}^{*}, i=1, \ldots, 8$, are calculable positive constants. Now, we select the $\epsilon_{i}$ 's such that $\epsilon_{1}+\ldots+\epsilon_{8}=1 / 2$. We then obtain

$$
\begin{gathered}
E_{\omega}(t, 0) \\
\leq 2 C_{1}^{*} \int_{0}^{t} \int_{R} \exp (-2 \omega s)\left(g_{s, i}+\phi g_{, i}\right)\left(g_{s, i}+\phi g_{, i}\right) d x d s+2 C_{2}^{*} \int_{0}^{t} \int_{R} \exp (-2 \omega s) l_{s, i} l_{s, i} d x d s \\
+2\left(C_{3}^{*}+C_{5}^{*}\right) \int_{0}^{t} \int_{R} \exp (-2 \omega s)\left(g_{s}+\phi g\right)^{2} d x d s+2 C_{4}^{*} \int_{0}^{t} \int_{R} \exp (-2 \omega s)\left(g_{s}+\phi g\right)^{q} d x d s \\
+2 C_{6}^{*} \int_{0}^{t} \int_{R} \exp (-2 \omega s) l_{s s}^{2} d x d s+2 C_{7}^{*} \int_{0}^{t} \int_{R} \exp (-2 \omega s) l_{s}^{2} d x d s \\
+2 C_{8}^{*} \int_{R} \exp (-2 \omega t) l_{t}^{2} d x .
\end{gathered}
$$

Furthermore,

$$
\begin{gathered}
\int_{0}^{t} \int_{R} \exp (-2 \omega s)\left(g_{s, i}+\phi g_{, i}\right)\left(g_{s, i}+\phi g_{, i}\right) d x d s \\
=\frac{1}{2 b} \int_{0}^{t} \int_{D} \exp (-2 \omega s)\left(h_{s, \alpha}+\phi h_{, \alpha}\right)\left(h_{s, \alpha}+\phi h_{, \alpha}\right) d a d s \\
+\frac{b}{2} \int_{0}^{t} \int_{D} \exp (-2 \omega s)\left(h_{s}+\phi h\right)\left(h_{s}+\phi h\right) d a d s,
\end{gathered}
$$

where, from now on, the index $\alpha$ only takes the values 2 and 3 , and

$$
\begin{gathered}
\int_{0}^{t} \int_{R} \exp (-2 \omega s) l_{s, i} l_{s, i} d x d s=\frac{1}{2 b} \int_{0}^{t} \int_{D} \exp (-2 \omega s) m_{s, \alpha} m_{s, \alpha} d a d s \\
+\frac{b}{2} \int_{0}^{t} \int_{D} \exp (-2 \omega s) m_{s}^{2} d a d s
\end{gathered}
$$




$$
\begin{aligned}
\int_{0}^{t} \int_{R} \exp (-2 \omega s)\left(g_{s}+\phi g\right)^{2} d x d s & =\frac{1}{2 b} \int_{0}^{t} \int_{D} \exp (-2 \omega s)\left(h_{s}+\phi h\right)^{2} d a d s \\
\int_{0}^{t} \int_{R} \exp (-2 \omega s)\left(g_{s}+\phi g\right)^{q} d x d s & =\frac{1}{q b} \int_{0}^{t} \int_{D} \exp (-2 \omega s)\left(h_{s}+\phi h\right)^{2} d a d s \\
\int_{0}^{t} \int_{R} \exp (-2 \omega s) l_{s s}^{2} d x d s & =\frac{1}{2 b} \int_{0}^{t} \int_{D} \exp (-2 \omega s) m_{s s}^{2} d a d s \\
\int_{0}^{t} \int_{R} \exp (-2 \omega s) l_{s}^{2} d x d s & =\frac{1}{2 b} \int_{0}^{t} \int_{D} \exp (-2 \omega s) m_{s}^{2} d a d s \\
\int_{R} \exp (-2 \omega s) l_{t}^{2} d x & =\frac{1}{2 b} \int_{D} \exp (-2 \omega s) m_{t}^{2} d a .
\end{aligned}
$$

We finally end up with the inequality

$$
\begin{gathered}
E_{\omega}(t, 0) \leq C_{1}^{*}\left(\frac{1}{b} \int_{0}^{t} \int_{D} \exp (-2 \omega s)\left(h_{s, \alpha}+\phi h_{, \alpha}\right)\left(h_{s, \alpha}+\phi h_{, \alpha}\right) d a d s\right. \\
\left.+b \int_{0}^{t} \int_{D} \exp (-2 \omega s)\left(h_{s}+\phi h\right)\left(h_{s}+\phi h\right) d a d s\right) \\
\left.+C_{2}^{*}\left(\frac{1}{b} \int_{0}^{t} \int_{D} \exp (-2 \omega s) m_{s, \alpha} m_{s, \alpha}\right) d a d s+b \int_{0}^{t} \int_{D} \exp (-2 \omega s) m_{s}^{2} d a d s\right) \\
+\frac{C_{4}^{*}}{q b} \int_{0}^{t} \int_{D} \exp (-2 \omega s)\left(h_{s}+\phi h\right)^{2} d a d s+\frac{C_{6}^{*}}{b} \int_{0}^{t} \int_{D}^{t} \exp (-2 \omega s) m_{s s}^{2} d a d s \\
\frac{C_{7}^{*}}{b} \int_{0}^{t} \int_{D} \exp (-2 \omega s) m_{s}^{2} d a d s+C_{8}^{*} b \int_{D} \exp (-2 \omega t) m_{t}^{2} d a .
\end{gathered}
$$

Here, we could optimize the right-hand side of this inequality with respect to the parameter $b$. However, this is not an easy task and we just consider the upper bound obtained for every given $b$.

Acknowledgments. This paper was initiated while A.M. was visiting the Universitat Politècnica de Catalunya in Terrassa. He wishes to thank this institution for its warm hospitality. R.Q. is supported by the Project "Análisis Matemático de las Ecuaciones en Derivada Parciales de la Termomecánica“ (MTM2013-42004-P) of the Spanish Ministry of Economy and Competitiveness. 


\section{REFERENCES}

[1] S. Aizicovici, E. Feireisl, Long-time stabilization of solutions to a phase-field model with memory, J. Evol. Eqns. 1 (2001), 69-84.

[2] S. Aizicovici, E. Feireisl, F. Issard-Roch, Long-time convergence of solutions to a phase-field system, Math. Methods Appl. Sci. 24 (2001), 277-287.

[3] D. Brochet, X. Chen, D. Hilhorst, Finite dimensional exponential attractors for the phase-field model, Appl. Anal. 49 (1993), 197-212.

[4] M. Brokate, J. Sprekels, Hysteresis and phase transitions, Springer, New York, 1996.

[5] G. Caginalp, An analysis of a phase field model of a free boundary, Arch. Rational Mech. Anal. 92 (1986), 205-245.

[6] P.J. Chen, M.E. Gurtin, On a theory of heat involving two temperatures, J. Appl. Math. Phys. (ZAMP) 19 (1968), 614-627.

[7] P.J. Chen, M.E. Gurtin, W.O. Williams, A note on non-simple heat conduction, J. Appl. Math. Phys. (ZAMP) 19 (1968), 969-970.

[8] P.J. Chen, M.E. Gurtin, W.O. Williams, On the thermodynamics of non-simple materials with two temperatures, J. Appl. Math. Phys. (ZAMP) 20 (1969), 107-112.

[9] L. Cherfils, A. Miranville, Some results on the asymptotic behavior of the Caginalp system with singular potentials, Adv. Math. Sci. Appl. 17 (2007), 107-129.

[10] L. Cherfils, A. Miranville, On the Caginalp system with dynamic boundary conditions and singular potentials, Appl. Math. 54 (2009), 89-115.

[11] R. Chill, E. Fašangovà, J. Prüss, Convergence to steady states of solutions of the Cahn-Hilliard equation with dynamic boundary conditions, Math. Nachr. 279 (2006), 1448-1462.

[12] M. Conti, S. Gatti, A. Miranville, A generalization of the Caginalp phase-field system with Neumann boundary conditions, Nonlinear Anal. 87 (2013), 11-21.

[13] C.I. Christov, P.M. Jordan, Heat conduction paradox involving second-sound propagation in moving media, Phys. Review Letters 94 (2005), 154301.

[14] B. Doumbé, Etude de modèles de champ de phase de type Caginalp, PhD thesis, Université de Poitiers, 2013.

[15] A.S. El-Karamany, M.A. Ezzat, On the two-temperature Green-Naghdi thermoelasticity theories, J. Thermal Stresses 34 (2011), 1207-1226.

[16] J.N. Flavin, R.J. Knops, L.E. Payne, Decay estimates for the constrained elastic cylinder of variable cross-section, Quart. Appl. Math. 47 (1989), 325-350.

[17] J.N. Flavin, R.J. Knops, L.E. Payne, Energy bounds in dynamical problems for a semi-infinite elastic beam, in "Elasticity: Mathematical Methods and Applications", G. Eason and R.W. Ogden eds., Chichester: Ellis Horwood, 101-111, 1989.

[18] C.G. Gal, M. Grasselli, The nonisothermal Allen-Cahn equation with dynamic boundary conditions, Discrete Contin. Dyn. Systems A 22 (2008), 1009-1040.

[19] P. Galenko, D. Jou, Diffuse-interface model for rapid phase transformations in nonequilibrium systems, Phys. Rev. E 71 (2005), 046125.

[20] S. Gatti, A. Miranville, Asymptotic behavior of a phase-field system with dynamic boundary conditions, in Differential equations: inverse and direct problems (Proceedings of the workshop "Evolution Equations: Inverse and Direct Problems", Cortona, June 21-25, 2004), A series of Lecture notes in pure and applied mathematics, Vol. 251, A. Favini and A. Lorenzi eds., Chapman \& Hall, 149-170, 2006.

[21] M. Grasselli, A. Miranville, V. Pata, S. Zelik, Well-posedness and long time behavior of a parabolichyperbolic phase-field system with singular potentials, Math. Nachr. 280 (2007), 1475-1509.

[22] M. Grasselli, A. Miranville, G. Schimperna, The Caginalp phase-field system with coupled dynamic boundary conditions and singular potentials, Discrete Contin. Dyn. Systems 28 (2010), 67-98.

[23] M. Grasselli, H. Petzeltová, G. Schimperna, Long time behavior of solutions to the Caginalp system with singular potential, Z. Anal. Anwend. 25 (2006), 51-72. 
[24] M. Grasselli, V. Pata, Existence of a universal attractor for a fully hyperbolic phase-field system, J. Evol. Eqns. 4 (2004), 27-51.

[25] A.E. Green, P.M. Naghdi, A re-examination of the basic postulates of thermomechanics, Proc. Royal Society London A 432 (1991), 171-194.

[26] C.O. Horgan, Recent developments concerning Saint-Venant's principle: A second update, Appl. Mech. Reviews 49 (1996), 101-111.

[27] C.O. Horgan, L.E. Payne, L.T. Wheeler, Spatial decay estimates in transient heat conduction, Quart. Appl. Math. 42 (1984), 119-127.

[28] C.O. Horgan, R. Quintanilla, Spatial decay of transient end effects in functionally graded heat conducting materials, Quart. Appl. Math. 59 (2001), 529-542.

[29] J. Jiang, Convergence to equilibrium for a parabolic-hyperbolic phase-field model with Cattaneo heat flux law, J. Math. Anal. Appl. 341 (2008), 149-169.

[30] J. Jiang, Convergence to equilibrium for a fully hyperbolic phase field model with Cattaneo heat flux law, Math. Methods Appl. Sci. 32 (2009), 1156-1182.

[31] A. Miranville, On a phase-field model with a logarithmic nonlinearity, Appl. Math. 57 (2012), 215229.

[32] A. Miranville, Some mathematical models in phase transition, Discrete Contin. Dyn. Systems Series S 7 (2014), 271-306.

[33] A. Miranville, R. Quintanilla, A generalization of the Caginalp phase-field system based on the Cattaneo law, Nonlinear Anal. TMA 71 (2009), 2278-2290.

[34] A. Miranville, R. Quintanilla, Some generalizations of the Caginalp phase-field system, Appl. Anal. 88 (2009), 877-894.

[35] A. Miranville, R. Quintanilla, A phase-field model based on a three-phase-lag heat conduction, Appl. Math. Optim. 63 (2011), 133-150.

[36] A. Miranville, R. Quintanilla, A type III phase-field system with a logarithmic potential, Appl. Math. Letters 24 (2011), 1003-1008.

[37] A. Miranville, R. Quintanilla, Spatial decay for several phase-field models, J. Appl. Math. Mech. (ZAMM) 93 (2013), 801-810.

[38] A. Miranville, R. Quintanilla, A generalization of the Allen-Cahn equation, IMA J. Appl. Math. 80 (2015), 410-430.

[39] A. Miranville, S. Zelik, Robust exponential attractors for singularly perturbed phase-field type equations, Electronic J. Diff. Eqns. 2002 (2002), 1-28.

[40] A. Miranville, S. Zelik, Robust exponential attractors for Cahn-Hilliard type equations with singular potentials, Math. Methods Appl. Sci. 27 (2004), 545-582.

[41] A. Miranville, S. Zelik, Attractors for dissipative partial differential equations in bounded and unbounded domains, in Handbook of Differential Equations, Evolutionary Partial Differential Equations, Vol. 4, C.M. Dafermos and M. Pokorny eds., Elsevier, Amsterdam, 103-200, 2008.

[42] R. Quintanilla, Damping of end effects in a thermoelastic theory, Appl. Math. Letters 14 (2001), $137-141$.

[43] R. Quintanilla, A well-posed problem for the three-dual-phase-lag heat conduction, J. Thermal Stresses 32 (2009), 1270-1278.

[44] R. Temam, Infinite-dimensional dynamical systems in mechanics and physics, Second edition, Applied Mathematical Sciences, Vol. 68, Springer-Verlag, New York, 1997.

[45] Z. Zhang, Asymptotic behavior of solutions to the phase-field equations with Neumann boundary conditions, Comm. Pure Appl. Anal. 4 (2005), 683-693.

\author{
${ }^{1}$ Université DE POITIERS \\ LABORAtoire de MathÉmatiques et Applications \\ UMR CNRS 7348 - SP2MI \\ Boulevard Marie et Pierre Curie - Téléport 2 \\ F-86962 Chasseneuil Futuroscope Cedex, France \\ E-mail address: Alain.Miranville@math.univ-poitiers.fr
}


${ }^{2}$ ETSEIAT-UPC

MAtemàtica Aplichda 2

Colom 11

S-08222 Terrassa, Barcelona, Spain

E-mail address: Ramon.Quintanilla@upc.edu 